\title{
Sumudu Decomposition Method for Solving Fractional Bratu-Type Differential Equations
}

\author{
N. B. Manjare*, H. T. Dinde \\ Department of Mathematics, Shivaji University, Kolhapur 416004. M.S., India
}

Received 17 May 2020, accepted in final revised form 27 June 2020

\begin{abstract}
The purpose of this paper is to introduce Sumudu decomposition method for solving Fractional Bratu-type differential equation. This method is a combination of the Sumudu transform and Adomian decomposition method. The fractional derivative is described in the Caputo sense. The Sumudu decomposition method is applied to obtain approximate analytical solution of non-linear Fractional Bratu-type differential equation. A novel combination of Sumudu transform and Adomian decomposition provides approximate solution in the form of infinite convergent series solution. The behavior of approximate analytical solutions and exact solutions for different values of $\alpha$ are plotted graphically. The results acquired from Sumudu decomposition method indicates that the proposed method is very well founded, suitable and effective. Finally, some numerical examples are given to illustrate the efficiency and applicability of our method.
\end{abstract}

Keywords: Fractional derivatives; Sumudu transform method; Adomian polynomials; Decomposition method; Fractional Bratu-type differential equations.

(C) 2020 JSR Publications. ISSN: 2070-0237 (Print); 2070-0245 (Online). All rights reserved. doi: http://dx.doi.org/10.3329/jsr.v12i4.47163

J. Sci. Res. 12 (4), 585-605 (2020)

\section{Introduction}

The mathematical models of various streams of science $\&$ engineering were developed by using fractional order differential equations. In recent years, Fractional Calculus has been used in many areas such as electrical networks, engineering, viscoelasticity, electrochemistry of corrosion [1], control theory of dynamical systems [2], biology [3], chemistry [4], physics [5], material science and signal processing [6]. The various contents of science and engineering can be successfully modeled by linear or nonlinear fractional order differential equations. The concept of Fractional Calculus and its applications were explained by Podlubny [1] and Kilbas [2].

Fractional Calculus has some essential differences in comparison with integer order [7]. The fractional order differential equations are in general form of integer order differential equations. The fractional derivatives are defined for whole time domain in physical process, whereas the integer order derivatives are connected to the local

\footnotetext{
${ }^{*}$ Corresponding author: nageshmanjare@gmail.com; drhtdmaths@gmail.com
} 
properties of a physical system at specific time. The author Saad along with other authors investigated different types of concepts in fractional calculus. The review of his research articles has been taken in brief for understanding history of fractional derivatives. The spectral collocation method based on the shifted Chebyshev polynomials has been applied for approximate solution of Korteweg-de Vries, Korteweg-de Vries-Burger's and Burger's equations with Liouville-Caputo fractional space derivatives, respectively [8]. A new model of fractional-order quadratic autocatalysis with linear inhibition was introduced by authors for its approximate solution using the power law, the exponential law and the Mittag-Leffler kernel [9]. The physical model called the blood ethanol concentration system, it can be expressed by a system of fractional differential equations (FDEs) has been solved by utilizing an approximate method for numerical solution. The authors have used the spectral collocation method based on Chebyshev approximations of the third-kind [10]. The approximate-analytical solutions obtained for a cancer chemotherapy effect model involving fractional derivatives with exponential kernel and with general Mittag-Leffler function. Laplace homotopy perturbation method and the modified homotopy analysis transform method were applied for approximate-analytical solutions [11]. The homotopy analysis transform method (HATM) was employed for solution of the time fractional order Korteweg-de Vries (KdV) and Korteweg-de VriesBurger's (KdVB) equations. The HATM is a combination of the Laplace decomposition method (LDM) and the homotopy analysis method (HAM) [12].

In the literature there are various kind of integral transforms have been used in physics and engineering. The integral transforms were successively applied to solve the differential equations, simultaneous linear equations, several works on the theory and application of integral transforms. The names of those transforms are Laplace Transform, Fourier Transform, Mellin Transform, Hankel Transform and Z Transform.

Watugala [13] introduced a new integral transform named as Sumudu transform. He applied such transform for solution of ordinary differential equations in control engineering problems. Various properties of Sumudu transform are observed in the literature [14].

The Sumudu transform is defined over the set of functions [6]

$$
\mathrm{A}=\left\{f(x): \exists M, \tau_{1}, \tau_{2}>0, f(x)<M e^{\frac{x}{\tau_{j}}}, \text { if } x \in(-1)^{j} \times[0, \infty)\right\}
$$

This is defined through definite integral by using the following formula:

$$
F(u)=S[f(x)]=\frac{1}{u} \int_{0}^{\infty} e^{\frac{-x}{u}} f(x) d x, u \in\left(-\tau_{1}, \tau_{2}\right) .
$$

When the exact solution of fractional order differential equations does not exist then many researchers particularly focus on approximate solutions of functional equations of fractional order. There are various numerical methods given for differential equations of integer order which are extended to fractional order differential equations, such methods are differential transform method (DTM) [15], variational iteration method (VIM) [16], Adomian decomposition method (ADM) [17], Homotopy perturbation method (HPM) 
[18], etc. This study motivated for us to focus on the solution of Bratu-type fractional differential equation [15].

$$
\begin{aligned}
& D^{\alpha} y(x)+\lambda e^{y(x)}=0, \quad 1<\alpha \leq 2,0<x<1 \\
& y(0)=a_{1}, y^{\prime}(0)=a_{2}
\end{aligned}
$$

Where $D^{\alpha}$ represents the fractional derivative of order $\alpha, y(x)$ is unknown function defined on the interval $[0,1]$ and $a_{1}, a_{2}$ and $\lambda$ are given constants. The numerical solutions of above-mentioned problem (2) have been previously calculated by ADM [19], RKM [20]. Further in this research area D. D. Demir and A. Zeybek obtained approximate solution of Fractional Bratu-type Differential Equation [FBDEs] by FDTM. In present paper we applied Sumudu decomposition method for approximate solution of problem (2). This technique has been developed and effectively used by different researchers [21-25]. The Adomian Decomposition method is useful for non-linear terms and Bratu-type fractional differential equation contain non-linear terms so as a result Sumudu decomposition method has given effective approximate solutions for different values of $\alpha$. Finally, approximate solutions of present method have compared with exact solution. Also, we compared approximate solutions of Sumudu decomposition method [SDM] with approximate solutions of fractional differential transform method [FDTM] \& fractional reproducing kernel Hilbert space method [FRKM]. The graphical representation shows the accuracy of the approximate and exact results.

This paper is divided in six sections: we have given introduction and literature review in section 1. We have provided some definitions of Fractional Calculus, definition of Sumudu transform and few properties of Sumudu transform in section 2. We have explained the fundamental theorems of convergence in Section 3. We have discussed analysis of Sumudu decomposition method in Section 4. We have solved some FBDEs for effectiveness of proposed method in section 5. Finally, section 6 concludes the paper.

\section{Basic of Fractional Calculus}

In this section, we introduce necessary definitions related to fractional calculus which are required in our present study.

\subsection{Definition [26]}

A real function $f(x), x>0$ is said to be in the space $C_{\eta}, \eta \in i$, if there exists a real number $p>n$, such that $f(x)=x^{p} g(x)$, where $g(x) \in C(0, \infty)$ and it is said to be in the space $C_{\eta}^{n}$ iff $f^{(n)} \in C_{\eta}, n \in N$.

\subsection{Definition [27]}

The Riemann-Liouville fractional differential operator of order $\alpha \geq 0$ of a function $f(t) \in \mu$ and $\mu \geq-1$ are defined by

$$
{ }_{a}^{R L} D_{x}^{\alpha} f(x)=\frac{1}{\Gamma(n-\alpha)} \frac{d^{n}}{d x^{n}} \int_{a}^{x} \frac{f(\xi)}{(x-\xi)^{\alpha+1-n}} d \xi ; n-1 \leq \alpha<n, n \in Z^{+}, x>a .
$$




\subsection{Definition [28]}

The fractional integral operator $\alpha \geq 0$ in the Riemann-Liouville sense of a function $f(x) \in C_{\eta}, \eta \geq-1$ is defined as

$$
J_{x}^{\alpha} f(x)=\frac{1}{\Gamma(\alpha)} \int_{0}^{x}(x-\xi)^{\alpha-1} f(\xi) d \xi ; \alpha>0, x>0 .
$$

\subsection{Definition [29]}

In the fractional calculus, the Caputo's fractional derivative is the definition which on the initial conditions gives most appropriate results for the initial conditions. The Caputo's fractional derivative is defined by

$$
{ }_{a}^{C} D_{x}^{\alpha} f(x)=\frac{1}{\Gamma(n-\alpha)} \int_{a}^{x} \frac{f^{(n)}(\xi)}{(x-\xi)^{\alpha+1-n}} d \xi ; n-1<\alpha<n .
$$

The Caputo's fractional derivative definition has one advantage; this is defined on initial conditions in same form with those of the equations of integer order.

Also, the Caputo's fractional derivative can be given by

$$
\begin{aligned}
& { }_{a}^{C} D^{\alpha} f(x)=I^{n-\alpha} \frac{d^{n}}{d x^{n}} f(x) ; \quad n-1<\alpha<n, \\
& { }_{a}^{C} D^{\alpha} f(x)=\frac{d^{n}}{d x^{n}} f(x) ; \quad \alpha=n .
\end{aligned}
$$

\subsection{Definition [30]}

The Sumudu transform $S\{f(x)\}$ of the fractional derivative introduced by Caputo is given by

$$
S\left\{D^{\alpha} f(x)\right\}=u^{-\alpha} G(u)-\sum_{k=0}^{n-1} u^{-\alpha+k} f^{(k)}(0), n-1<\alpha \leq n, \text { Where } G(u)=S\{f(x)\} .
$$

Many of special properties of the Sumudu transform are mentioned and tabulated in [30]. Some special properties of the Sumudu transform are as follows;

(i) $S\{1\}=1$

(ii) $S\left\{x^{n}\right\}=u^{n} \Gamma(\mathrm{n}+1), \quad n>0$

(iii) $S\{f(x) \pm g(x)\}=S\{f(x)\} \pm S\{g(x)\}$

\section{Fundamental Theorems of Convergence}

\subsection{Theorem}

A necessary condition for convergence of an infinite series $\sum_{n=0}^{\infty} u_{n}$ is that $\lim _{n \rightarrow \infty} u_{n}=$ 0 .

\subsection{Theorem}

A necessary condition for convergence of positive term series is defined follows (Pringsheim's Theorem). 
Statement: If a series $\sum_{n=0}^{\infty} u_{n}$ of positive monotonic decreasing terms converges then not only $u_{n} \rightarrow 0$ but also $n u_{n} \rightarrow 0$ as $n \rightarrow \infty$.

\subsection{Taylor's theorem}

Statement: If $f:[a, b] \rightarrow R$ is (n+1) times continuously differentiable, then there exits $c \in(a, b)$ such that

$$
\begin{aligned}
& f(b)=f(a)+f^{\prime}(a)(b-a)+\frac{f^{\prime \prime}(a)}{2}(b-a)^{2}+\cdots \ldots+\frac{f^{n}(a)}{n !}(b-a)^{n}+\frac{f^{(n+1)}(c(x))}{(n+1) !}(b- \\
& a)^{(n+1)} .
\end{aligned}
$$

The Taylor Theorem is usually applied for a fixed point $a$, while the point $b=x$ is used as an independent variable:

$$
f(x)=f(a)+f^{\prime}(a)(x-a)+\frac{f^{\prime \prime}(a)}{2}(x-a)^{2}+\cdots \ldots+\frac{f^{n}(a)}{n !}(x-a)^{n}+R_{n}(x) .
$$

where the remainder function $R_{n}(x)$ is given by

$$
R_{n}(x)=\frac{f^{(n+1)}(c(x))}{(n+1) !}(x-a)^{(n+1)} \text { with } c \in(a, x) .
$$

\subsection{Corollary of Taylors theorem}

Let $f: D \rightarrow R$ be infinitely differentiable with Taylor polynomials $T_{n}$ and remainders $R_{n}$, that is, for $n \geq 1$ holds

$$
f(x)=T_{n}(x)+R_{n}(x) .
$$

If $R_{n}(x) \rightarrow 0$ as $n \rightarrow \infty$ for $x \in D$, then the Taylor series centered at $x=a$ converges on $D$ to the function values $f(x)$, that is $f(x)=\sum_{n=0}^{\infty} \frac{f^{n}(a)}{n !}(x-a)^{n}$.

\section{Analysis of the Method [SDM]}

The fractional differential equation of the form

$$
D^{\alpha} y(x)+R(y)+N(x-\tau)=f(x) \quad \tau \in R, x<\tau, \quad n-1<\alpha \leq n,
$$

With initial condition

$$
u^{k}(0)=u_{0}^{k}
$$

where $R$ is a linear bounded operator and $N$ is a nonlinear bounded operator, $f(x)$ is a given continuous function and $D^{\alpha} y(x)$ is the term of the fractional order derivative.

In proposed article we applied same methodology of Sumudu decomposition method for analytical approximate series solution of (2) subject to initial conditions along with exclusion of linear bounded operator $R$ and exclusion of continuous function $\mathrm{f}(\mathrm{x})$ because the Bratu-type differential equation does not contain linear term and function of $\mathrm{x}$.

In this paper we will consider a class of Bratu-type differential equation of the form

$$
D^{\alpha} y(x)+N(x-\tau)=0 \quad \tau \in R, x<\tau, \quad n-1<\alpha \leq n,
$$


With initial condition

$$
u^{k}(0)=u_{0}^{k}
$$

Where $N$ is a nonlinear bounded operator and $D^{\alpha} y(x)$ is the term of the fractional order derivative (in throughout paper $R(y)$ and $f(x)$ both are absent).

The Sumudu decomposition method consists of applying the Sumudu transform first on both sides of (9) to give

$$
S\left\{D^{\alpha} y(x)\right\}+S\{N(x-\tau)\}=0
$$

By definition 2.5,

$$
\begin{aligned}
& \frac{S\{y(x)\}}{u^{\alpha}}-\frac{C}{u^{\alpha-K}}+S\{N(x-\tau)\}=0 \text { where } C=\sum_{k=0}^{n-1} f^{(k)}(0) \\
& S\{y(x)\}=u^{k} C-u^{\alpha} S\{N(x-\tau)\} .
\end{aligned}
$$

The standard Sumudu decomposition method defines the solution $y(x)$ by the series

$$
y(x)=\sum_{n=0}^{\infty} y_{n}(x)
$$

Also, the non -linear operator is decomposed as $N(x-\tau)=\sum_{n=0}^{\infty} A_{n}$.

Where $A_{n}$ i.e. Adomian Polynomials of $y_{0}, y_{1}, y_{2}, \ldots \ldots, y_{n}$ that are given by:

$$
A_{n}=\frac{1}{n !} \frac{d^{n}}{d \lambda^{n}}\left[N\left(\sum_{n=0}^{\infty} \lambda^{n} y_{n}\right)\right]_{\lambda=0}, n=0,1,2, \ldots \ldots \ldots
$$

The first Adomian Polynomials are given by

$$
\begin{aligned}
& A_{0}=N\left(y_{0}\right) \\
& A_{1}=y_{1} N^{\prime}\left(y_{0}\right) \\
& A_{2}=y_{2} N^{\prime}\left(y_{0}\right)+\frac{1}{2 !} y_{1}^{2} N^{\prime \prime}\left(y_{0}\right) \\
& A_{3}=y_{3} N^{\prime}\left(y_{0}\right)+y_{1} y_{2} N^{\prime \prime}\left(y_{0}\right)+\frac{1}{3 !} y_{1}^{3} N^{\prime \prime \prime}\left(y_{0}\right)
\end{aligned}
$$

We apply (12) and (13) in (11) we get,

$$
S\left\{\sum_{n=0}^{\infty} y_{n}\right\}=u^{k} C-u^{\alpha} S\left\{\sum_{n=0}^{\infty} A_{n}\right\}
$$

Comparing both sides of (15)

$$
\begin{aligned}
& S\left\{y_{0}\right\}=u^{k} C, \\
& S\left\{y_{1}\right\}=-u^{\alpha} S\left\{A_{0}\right\}, \\
& S\left\{y_{2}\right\}=-u^{\alpha} S\left\{A_{1}\right\} .
\end{aligned}
$$

In general, the recursive relation is given by

$$
S\left\{y_{n}\right\}=-u^{\alpha} S\left\{A_{n-1}\right\}, \quad n \geq 1,
$$

Further we apply inverse Sumudu transform to (16)-(19) then: 


$$
\begin{aligned}
& y_{0}=F(x), \\
& y_{n}=-S^{-1}\left[u^{\alpha} S\left\{A_{n-1}\right\}\right], \quad n \geq 1 .
\end{aligned}
$$

Where $F(x)$ is a function that arises from the source term and prescribed initial conditions.

\section{Some Numerical Examples}

\subsection{Example}

We introduce the following Bratu-type differential equation of fractional order

$$
D^{\alpha} y(x)-2 e^{y(x)}=0, \quad 1<\alpha \leq 2, \quad 0<x<1,
$$

Subject to initial condition

$$
y(0)=0, y^{\prime}(0)=0
$$

The exact solution is given by $y(x)=-2 \ln (\cos x)$ for $\alpha=2$.

For approximate solution, we apply Sumudu transform to both side of equation (22)

$$
\begin{aligned}
& S\left\{D^{\alpha} y(x)\right\}-2 S\left\{e^{y(x)}\right\}=0 \\
& S\left\{D^{\alpha} y(x)\right\}=2 S\left\{e^{y(x)}\right\}
\end{aligned}
$$

By using definition 2.5 and initial condition (23) we have:

$$
\begin{aligned}
& \frac{Y(u)}{u^{\alpha}}-\frac{y(0)}{u^{\alpha-0}}-\frac{y^{\prime}(0)}{u^{\alpha-1}}=2 S\left\{e^{y(x)}\right\} \\
& \frac{Y(u)}{u^{\alpha}}-\frac{0}{u^{\alpha-0}}-\frac{0}{u^{\alpha-1}}=2 S\left\{e^{y(x)}\right\} \\
& \frac{Y(u)}{u^{\alpha}}=2 S\left\{e^{y(x)}\right\} \\
& Y(u)=2 u^{\alpha} S\left\{e^{y(x)}\right\} \\
& S\{y(x)\}=2 u^{\alpha} S\left\{e^{y(x)}\right\}
\end{aligned}
$$

Further we apply inverse Sumudu transform to (24) to get

$$
\begin{aligned}
& y(x)=S^{-1}\left[2 u^{\alpha} S\left\{e^{y(x)}\right\}\right] \\
& =2 S^{-1}\left[u^{\alpha} S\left\{e^{y(x)}\right\}\right] \\
& y_{0}(x)=S^{-1}\{0\}=0 \\
& y_{n+1}(x)=2 S^{-1}\left[u^{\alpha} S\left\{A_{n}\right\}\right]
\end{aligned}
$$

In this example, the nonlinear term is $N=e^{y(x)}$

From equation (14) we have

$$
A_{0}=e^{y_{0}(x)}
$$




$$
\begin{aligned}
& A_{1}=y_{1}(x) e^{y_{0}(x)} \\
& A_{2}=y_{2}(x) e^{y_{0}(x)}+\frac{y_{1}^{2}(x)}{2 !} e^{y_{0}(x)}
\end{aligned}
$$

We put $n=0$ in equation (26)

$$
y_{1}(x)=2 S^{-1}\left[u^{\alpha} S\left\{A_{0}\right\}\right]
$$

Substituting equation (27) in (28) we get

$$
\begin{aligned}
y_{1}(x) & =2 S^{-1}\left[u^{\alpha} S\left\{e^{y_{0}(x)}\right\}\right] \\
& =2 S^{-1}\left[u^{\alpha} S\left\{e^{0}\right\}\right] \\
& =2 S^{-1}\left[u^{\alpha} S\{1\}\right] \\
& =2 S^{-1}\left[u^{\alpha}\right] \text { where } S\{1\}=1 \\
y_{1}(x) & =\frac{2 x^{\alpha}}{\Gamma(\alpha+1)}
\end{aligned}
$$

We put $n=1$ in equation (26)

$$
y_{2}(x)=2 S^{-1}\left[u^{\alpha} S\left\{A_{1}\right\}\right]
$$

Substituting equation (27) in (29) we get

$$
\begin{aligned}
y_{2}(x) & =2 S^{-1}\left[u^{\alpha} S\left\{y_{1}(x) e^{y_{0}(x)}\right\}\right] \\
& =2 S^{-1}\left[u^{\alpha} S\left\{\frac{2 x^{\alpha}}{\Gamma(\alpha+1)} e^{0}\right\}\right] \\
& =2 S^{-1}\left[u^{\alpha} S\left\{\frac{2 x^{\alpha}}{\Gamma(\alpha+1)} 1\right\}\right] \\
& =2 S^{-1}\left[u^{\alpha} S\left\{\frac{2 x^{\alpha}}{\Gamma(\alpha+1)}\right\}\right] \\
& =2 S^{-1}\left[u^{\alpha} \frac{2}{\Gamma(\alpha+1)} S\left\{x^{\alpha}\right\}\right] \\
& =2 S^{-1}\left[\frac{2 u^{\alpha}}{\Gamma(\alpha+1)} S\left\{x^{\alpha}\right\}\right] \\
& =2 S^{-1}\left[\frac{2 u^{\alpha}}{\Gamma(\alpha+1)} u^{\alpha} \Gamma(\alpha+1)\right] \\
& =4 S^{-1}\left[u^{2 \alpha}\right] \\
y_{2}(x) & =\frac{4 x^{2 \alpha}}{\Gamma(2 \alpha+1)}
\end{aligned}
$$

We put $n=2$ in equation (26)

$$
y_{3}(x)=2 S^{-1}\left[u^{\alpha} S\left\{A_{2}\right\}\right]
$$

Substituting equation (27) in (30) we get 


$$
\begin{aligned}
y_{3}(x) & =2 S^{-1}\left[u^{\alpha} S\left\{y_{2}(x) e^{y_{0}(x)}+\frac{y_{1}^{2}(x)}{2 !} e^{y_{0}(x)}\right\}\right] \\
& =2 S^{-1}\left[u^{\alpha} S\left\{\frac{4 x^{2 \alpha}}{\Gamma(2 \alpha+1)} e^{0}+\frac{\left(\frac{2 x^{\alpha}}{\Gamma(\alpha+1)}\right)^{2}}{2 !} e^{0}\right\}\right] \\
& =2 S^{-1}\left[u^{\alpha} S\left\{\frac{4 x^{2 \alpha}}{\Gamma(2 \alpha+1)} 1+\frac{4 x^{2 \alpha}}{2(\Gamma(\alpha+1))^{2}} 1\right\}\right] \\
& =8 S^{-1}\left[u^{\alpha} \frac{\left[2(\Gamma(\alpha+1))^{2}+\Gamma(2 \alpha+1)\right]}{2 \Gamma(2 \alpha+1)(\Gamma(\alpha+1))^{2}} S\left\{x^{2 \alpha}\right\}\right] \\
& =8 S^{-1}\left[u^{\alpha} \frac{\left[2(\Gamma(\alpha+1))^{2}+\Gamma(2 \alpha+1)\right]}{2 \Gamma(2 \alpha+1)(\Gamma(\alpha+1))^{2}} u^{2 \alpha} \Gamma(2 \alpha+1)\right] \\
& =8 \frac{\left[2(\Gamma(\alpha+1))^{2}+\Gamma(2 \alpha+1)\right]}{2(\Gamma(\alpha+1))^{2}} S^{-1}\left[u^{3 \alpha}\right] \\
& =4 \frac{\left[2(\Gamma(\alpha+1))^{2}+\Gamma(2 \alpha+1)\right]}{(\Gamma(\alpha+1))^{2}} \frac{x^{3 \alpha}}{\Gamma(3 \alpha+1)} \\
y_{3}(x) & =4 \frac{\left[2(\Gamma(\alpha+1))^{2}+\Gamma(2 \alpha+1)\right]}{\Gamma(3 \alpha+1)(\Gamma(\alpha+1))^{2}} x^{3 \alpha}
\end{aligned}
$$

The series solution is given by

$$
\begin{aligned}
y(x) & =y_{0}(x)+y_{1}(x)+y_{2}(x)+y_{3}(x) \ldots \ldots \ldots \ldots \\
& =0+\frac{2 x^{\alpha}}{\Gamma(\alpha+1)}+\frac{4 x^{2 \alpha}}{\Gamma(2 \alpha+1)}+4 \frac{\left[2(\Gamma(\alpha+1))^{2}+\Gamma(2 \alpha+1)\right]}{\Gamma(3 \alpha+1)(\Gamma(\alpha+1))^{2}} x^{3 \alpha}+\cdots
\end{aligned}
$$

In particular case $\alpha=2$ then we get

$$
y(x)=x^{2}+\frac{x^{4}}{6}+\frac{2}{45} x^{6}+\cdots
$$

Table 1 shows that approximate solutions of y(t) for (22) obtained for different values of $\alpha$ using the Sumudu decomposition method, DTM [15] and RKM [20]. We can observe the numerical results in Table 1, it is clear that the approximate solutions of SDM are in best agreement with approximate solutions of DTM and RKM. Those solutions are constantly based on the Caputo fractional derivative. According to Taylors Theorem and its corollary, the obtained Taylor series is convergent about $\mathrm{a}=0$.

Fig. 1 shows the behaviour of approximate solutions versus exact solution. The nonlinear differential equation plots non-linear curves for various approximate values $\alpha=0.5,0.6,0.7,0.8,0.9$ and exact value $\alpha=1$. All non-linear curves of approximate solutions are very close to non-linear curve of exact solution.

Table 1. The comparisons of SDM, DTM and RKM for various values of $\alpha$. (for $\mathrm{x}=0.1$ and $\mathrm{N}=\mathrm{n}=5$ ).

\begin{tabular}{cccc}
\hline$\alpha$ & SDM & DTM [15] & RKM [20] \\
\hline 1.5 & $4.7780 \mathrm{E}-2$ & $4.8260 \mathrm{E}-2$ & $3.7921 \mathrm{E}-2$ \\
1.6 & $3.5470 \mathrm{E}-2$ & $3.5472 \mathrm{E}-2$ & $2.9221 \mathrm{E}-2$ \\
1.7 & $2.5988 \mathrm{E}-2$ & $2.5993 \mathrm{E}-2$ & $2.2504 \mathrm{E}-2$ \\
1.8 & $1.8975 \mathrm{E}-2$ & $1.8983 \mathrm{E}-2$ & $1.7221 \mathrm{E}-2$ \\
1.9 & $1.3814 \mathrm{E}-2$ & $1.3814 \mathrm{E}-2$ & $1.3082 \mathrm{E}-2$ \\
\hline
\end{tabular}




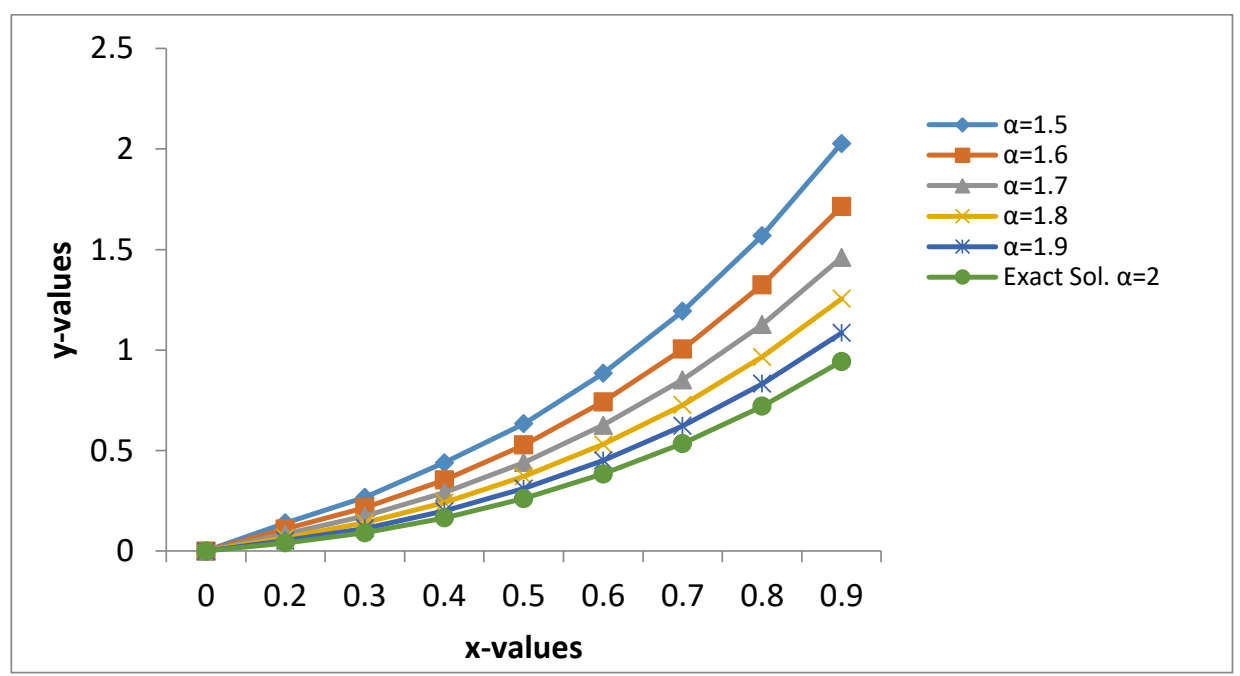

Fig. 1. The behavior of approximate solutions for the various values of $\alpha$ versus exact solution.

\subsection{Example}

Consider the following problem

$$
D^{\alpha} y(x)-e^{2 y(x)}=0, \quad 1<\alpha \leq 2, \quad 0<x<1,
$$

Subject to initial condition

$$
y(0)=0, y^{\prime}(0)=0
$$

The exact solution is given by $y(x)=\ln (\sec x)$ for $\alpha=2$.

For approximate solution, we apply Sumudu transform to both side of equation (31)

$$
\begin{aligned}
& S\left\{D^{\alpha} y(x)\right\}-S\left\{e^{2 y(x)}\right\}=0 \\
& S\left\{D^{\alpha} y(x)\right\}=S\left\{e^{2 y(x)}\right\}
\end{aligned}
$$

By using definition 2.5 and initial condition (32) we have:

$$
\begin{aligned}
& \frac{Y(u)}{u^{\alpha}}-\frac{y(0)}{u^{\alpha-0}}-\frac{y^{\prime}(0)}{u^{\alpha-1}}=S\left\{e^{2 y(x)}\right\} \\
& \frac{Y(u)}{u^{\alpha}}-\frac{0}{u^{\alpha-0}}-\frac{0}{u^{\alpha-1}}=S\left\{e^{2 y(x)}\right\} \\
& \frac{Y(u)}{u^{\alpha}}=S\left\{e^{2 y(x)}\right\} \\
& Y(u)=u^{\alpha} S\left\{e^{2 y(x)}\right\} \\
& S\{y(x)\}=u^{\alpha} S\left\{e^{2 y(x)}\right\}
\end{aligned}
$$

Further we apply inverse Sumudu transform to (33) to get 


$$
\begin{aligned}
& y(x)=S^{-1}\left[u^{\alpha} S\left\{e^{2 y(x)}\right\}\right] \\
& =S^{-1}\left[u^{\alpha} S\left\{e^{2 y(x)}\right\}\right] \\
& y_{0}(x)=S^{-1}\{0\}=0 \\
& y_{n+1}(x)=S^{-1}\left[u^{\alpha} S\left\{A_{n}\right\}\right]
\end{aligned}
$$

In this example, the nonlinear term is $N=e^{2 y(x)}$

From equation (14) we have

$$
\begin{aligned}
& A_{0}=e^{2 y_{0}(x)} \\
& A_{1}=2 y_{1}(x) e^{2 y_{0}(x)} \\
& A_{2}=2 y_{2}(x) e^{2 y_{0}(x)}+2 y_{1}^{2}(x) e^{2 y_{0}(x)}
\end{aligned}
$$

We put $n=0$ in equation (35)

$$
y_{1}(x)=S^{-1}\left[u^{\alpha} S\left\{A_{0}\right\}\right]
$$

Substituting equation (36) in (37) we get

$$
\begin{aligned}
y_{1}(x) & =S^{-1}\left[u^{\alpha} S\left\{e^{2 y_{0}(x)}\right\}\right] \\
& =S^{-1}\left[u^{\alpha} S\left\{e^{0}\right\}\right] \\
& =S^{-1}\left[u^{\alpha} S\{1\}\right] \\
& =S^{-1}\left[u^{\alpha}\right] \text { where } S\{1\}=1 \\
y_{1}(x) & =\frac{x^{\alpha}}{\Gamma(\alpha+1)}
\end{aligned}
$$

We put $n=1$ in equation (35)

$$
y_{2}(x)=S^{-1}\left[u^{\alpha} S\left\{A_{1}\right\}\right]
$$

Substituting equation (36) in (38) we get

$$
\begin{aligned}
y_{2}(x) & =S^{-1}\left[u^{\alpha} S\left\{2 y_{1}(x) e^{2 y_{0}(x)}\right\}\right] \\
& =S^{-1}\left[u^{\alpha} S\left\{2 \frac{x^{\alpha}}{\Gamma(\alpha+1)} e^{0}\right\}\right] \\
& =S^{-1}\left[u^{\alpha} S\left\{2 \frac{x^{\alpha}}{\Gamma(\alpha+1)} 1\right\}\right] \\
& =S^{-1}\left[u^{\alpha} S\left\{2 \frac{x^{\alpha}}{\Gamma(\alpha+1)}\right\}\right] \\
& =S^{-1}\left[u^{\alpha} \frac{2}{\Gamma(\alpha+1)} S\left\{x^{\alpha}\right\}\right] \\
& =S^{-1}\left[\frac{2 u^{\alpha}}{\Gamma(\alpha+1)} S\left\{x^{\alpha}\right\}\right] \\
& =S^{-1}\left[\frac{2 u^{\alpha}}{\Gamma(\alpha+1)} u^{\alpha} \Gamma(\alpha+1)\right]
\end{aligned}
$$




$$
\begin{aligned}
& =2 S^{-1}\left[u^{2 \alpha}\right] \\
y_{2}(x) & =\frac{2 x^{2 \alpha}}{\Gamma(2 \alpha+1)}
\end{aligned}
$$

We put $n=2$ in equation (35)

$$
y_{3}(x)=S^{-1}\left[u^{\alpha} S\left\{A_{2}\right\}\right]
$$

Substituting equation (36) in (39) we get

$$
\begin{aligned}
y_{3}(x) & =S^{-1}\left[u^{\alpha} S\left\{2 y_{2}(x) e^{2 y_{0}(x)}+2 y_{1}^{2}(x) e^{2 y_{0}(x)}\right\}\right] \\
& =S^{-1}\left[u^{\alpha} S\left\{2 \frac{2 x^{2 \alpha}}{\Gamma(2 \alpha+1)} e^{0}+2\left(\frac{x^{\alpha}}{\Gamma(\alpha+1)}\right)^{2} e^{0}\right\}\right] \\
& =S^{-1}\left[u^{\alpha} S\left\{2 \frac{2 x^{2 \alpha}}{\Gamma(2 \alpha+1)} 1+2 \frac{x^{2 \alpha}}{(\Gamma(\alpha+1))^{2}} 1\right\}\right] \\
& =S^{-1}\left[2 u^{\alpha} \frac{\left[2(\Gamma(\alpha+1))^{2}+\Gamma(2 \alpha+1)\right]}{\Gamma(2 \alpha+1)(\Gamma(\alpha+1))^{2}} S\left\{x^{2 \alpha}\right\}\right] \\
& =S^{-1}\left[2 u^{\alpha} \frac{\left[2(\Gamma(\alpha+1))^{2}+\Gamma(2 \alpha+1)\right]}{\Gamma(2 \alpha+1)(\Gamma(\alpha+1))^{2}} u^{2 \alpha} \Gamma(2 \alpha+1)\right] \\
& =2 \frac{\left[2(\Gamma(\alpha+1))^{2}+\Gamma(2 \alpha+1)\right]}{(\Gamma(\alpha+1))^{2}} S^{-1}\left[u^{3 \alpha}\right] \\
& =2 \frac{\left[2(\Gamma(\alpha+1))^{2}+\Gamma(2 \alpha+1)\right]}{(\Gamma(\alpha+1))^{2}} \frac{x^{3 \alpha}}{\Gamma(3 \alpha+1)} \\
y_{3}(x) & =2 \frac{\left[2(\Gamma(\alpha+1))^{2}+\Gamma(2 \alpha+1)\right]}{\Gamma(3 \alpha+1)(\Gamma(\alpha+1))^{2}} x^{3 \alpha}
\end{aligned}
$$

The series solution is given by

$$
\begin{aligned}
y(x) & =y_{0}(x)+y_{1}(x)+y_{2}(x)+y_{3}(x) \ldots \ldots \ldots \ldots \\
& =0+\frac{x^{\alpha}}{\Gamma(\alpha+1)}+\frac{2 x^{2 \alpha}}{\Gamma(2 \alpha+1)}+2 \frac{\left[2(\Gamma(\alpha+1))^{2}+\Gamma(2 \alpha+1)\right]}{\Gamma(3 \alpha+1)(\Gamma(\alpha+1))^{2}} x^{3 \alpha}+\cdots
\end{aligned}
$$

In particular case $\alpha=2$ then we get

$$
y(x)=\frac{x^{2}}{2 !}+\frac{2 x^{4}}{4 !}+\frac{16}{6 !} x^{6}+\cdots
$$

Table 2 shows that approximate solutions of $y(t)$ for (31) obtained for different values of $\alpha$ using the Sumudu decomposition method, DTM [15] and RKM [20]. We can observe the numerical results in Table 2, it is clear that the approximate solutions of SDM are in best agreement with approximate solutions of DTM and RKM. Those solutions are constantly based on the Caputo fractional derivative. According to Taylors Theorem and its corollary, the obtained Taylor series is convergent about $\mathrm{a}=0$.

Fig. 1 shows the behaviour of approximate solutions versus exact solution. The nonlinear differential equation plots non-linear curves for various approximate values $\alpha=0.5,0.6,0.7,0.8,0.9$ and exact value $\alpha=1$. All non-linear curves of approximate solutions are very close to non-linear curve of exact solution. 
Table 2. The comparisons of SDM, DTM and RKM for various values of $\alpha$. (for $\mathrm{x}=0.1$ and $\mathrm{N}=\mathrm{n}=5$ ).

\begin{tabular}{cccc}
\hline$\alpha$ & SDM & DTM [15] & RKM [20] \\
\hline 1.5 & $2.4130 \mathrm{E}-2$ & $2.3957 \mathrm{E}-2$ & $2.3791 \mathrm{E}-2$ \\
1.6 & $1.7735 \mathrm{E}-2$ & $1.7652 \mathrm{E}-2$ & $1.7571 \mathrm{E}-2$ \\
1.7 & $1.2996 \mathrm{E}-2$ & $1.2956 \mathrm{E}-2$ & $1.2917 \mathrm{E}-2$ \\
1.8 & $9.4913 \mathrm{E}-3$ & $9.4725 \mathrm{E}-3$ & $9.4537 \mathrm{E}-3$ \\
1.9 & $6.9070 \mathrm{E}-3$ & $6.8982 \mathrm{E}-3$ & $6.8894 \mathrm{E}-3$ \\
\hline
\end{tabular}

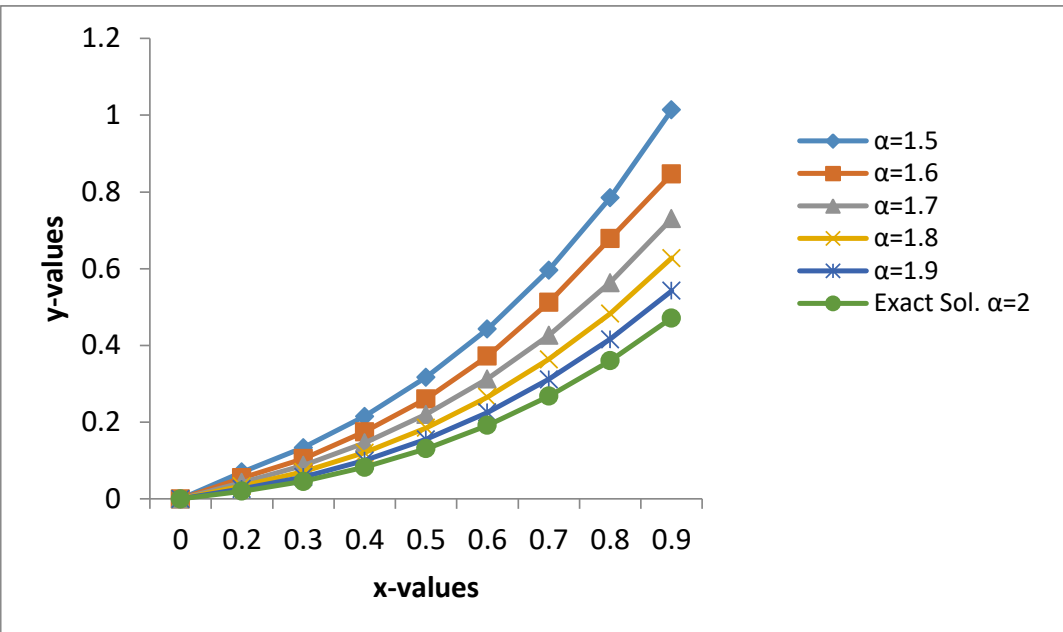

Fig. 2. The behavior of approximate solutions for the various values of $\alpha$ versus exact solution.

\subsection{Example}

Consider the following Bratu-type problem

$$
D^{2 \alpha} y(x)-\pi^{2} e^{y(x)}=0, \quad 0<\alpha \leq 2, \quad 0<x<1,
$$

Subject to initial condition

$$
y(0)=0, y^{\alpha}(0)=\pi
$$

The exact solution is given by $y(x)=-\ln (1-\sin (\pi x))$ for $\alpha=2$.

For approximate solution, we apply Sumudu transform to both side of equation (40)

$$
\begin{aligned}
& S\left\{D^{2 \alpha} y(x)\right\}-\pi^{2} S\left\{e^{y(x)}\right\}=0 \\
& S\left\{D^{2 \alpha} y(x)\right\}=\pi^{2} S\left\{e^{y(x)}\right\}
\end{aligned}
$$

By using definition 2.5 and initial condition (41) we have:

$$
\begin{aligned}
& \frac{Y(u)}{u^{2 \alpha}}-\frac{y(0)}{u^{2 \alpha-0}}-\frac{y^{\alpha}(0)}{u^{2 \alpha-\alpha}}=\pi^{2} S\left\{e^{y(x)}\right\} \\
& \frac{Y(u)}{u^{2 \alpha}}-\frac{\pi}{u^{\alpha}}=\pi^{2} S\left\{e^{y(x)}\right\}
\end{aligned}
$$




$$
\begin{aligned}
& Y(u)=\pi u^{\alpha}+\pi^{2} u^{2 \alpha} S\left\{e^{y(x)}\right\} \\
& S\{y(x)\}=\pi u^{\alpha}+\pi^{2} u^{2 \alpha} S\left\{e^{y(x)}\right\}
\end{aligned}
$$

Further we apply inverse Sumudu transform to (42) to get

$$
\begin{aligned}
y(x) & =S^{-1}\left[\pi u^{\alpha}+\pi^{2} u^{2 \alpha} S\left\{e^{y(x)}\right\}\right], \\
& =\pi S^{-1}\left[u^{\alpha}\right]+\pi^{2} S^{-1}\left[u^{2 \alpha} S\left\{e^{y(x)}\right\}\right]
\end{aligned}
$$

Applying the Sumudu transform to initial condition yields $y_{0}(0)=0$. Considering $y_{1}(x)$ from source term and prescribed initial conditions

$$
\begin{aligned}
& y_{1}(x)=\pi S^{-1}\left[u^{\alpha}\right]=\frac{\pi x^{\alpha}}{\Gamma(\alpha+1)} \\
& y_{n+2}(x)=\pi^{2} S^{-1}\left[u^{2 \alpha} S\left\{A_{n}\right\}\right]
\end{aligned}
$$

In this example, the nonlinear term is $N=e^{y(x)}$

From equation (14) we have

$$
\begin{aligned}
& A_{0}=e^{y_{0}(x)} \\
& A_{1}=y_{1}(x) e^{y_{0}(x)} \\
& A_{2}=y_{2}(x) e^{y_{0}(x)}+\frac{y_{1}^{2}(x)}{2 !} e^{y_{0}(x)}
\end{aligned}
$$

We put $n=0$ in equation (44)

$$
y_{2}(x)=\pi^{2} S^{-1}\left[u^{2 \alpha} S\left\{A_{0}\right\}\right]
$$

Substituting equation (45) in (46) we get

$$
\begin{aligned}
y_{2}(x) & =\pi^{2} S^{-1}\left[u^{2 \alpha} S\left\{e^{y_{0}(x)}\right\}\right] \\
& =\pi^{2} S^{-1}\left[u^{2 \alpha} S\left\{e^{0}\right\}\right] \\
& =\pi^{2} S^{-1}\left[u^{2 \alpha} S\{1\}\right] \\
& =\pi^{2} S^{-1}\left[u^{2 \alpha}\right] \text { where } S\{1\}=1 \\
y_{2}(x) & =\frac{\pi^{2} x^{2 \alpha}}{\Gamma(2 \alpha+1)}
\end{aligned}
$$

We put $n=1$ in equation (44)

$$
y_{3}(x)=\pi^{2} S^{-1}\left[u^{2 \alpha} S\left\{A_{1}\right\}\right]
$$

Substituting equation (45) in (47) we get

$$
\begin{aligned}
y_{3}(x) & =\pi^{2} S^{-1}\left[u^{2 \alpha} S\left\{y_{1}(x) e^{y_{0}(x)}\right\}\right] \\
& =\pi^{2} S^{-1}\left[u^{2 \alpha} S\left\{\frac{\pi x^{\alpha}}{\Gamma(\alpha+1)} e^{y_{0}(x)}\right\}\right] \\
& =\pi^{2} S^{-1}\left[u^{2 \alpha} S\left\{\frac{\pi x^{\alpha}}{\Gamma(\alpha+1)} e^{0}\right\}\right]
\end{aligned}
$$




$$
\begin{aligned}
& =\pi^{2} S^{-1}\left[u^{2 \alpha} S\left\{\frac{\pi x^{\alpha}}{\Gamma(\alpha+1)} 1\right\}\right] \\
& =\pi^{2} S^{-1}\left[u^{2 \alpha} \frac{\pi}{\Gamma(\alpha+1)} S\left\{x^{\alpha}\right\}\right] \\
& =\pi^{2} S^{-1}\left[u^{2 \alpha} \frac{\pi}{\Gamma(\alpha+1)} u^{\alpha} \Gamma(\alpha+1)\right] \\
& =\pi^{3} S^{-1}\left[u^{3 \alpha}\right] \\
y_{3}(x) & =\frac{\pi^{3} x^{3 \alpha}}{\Gamma(3 \alpha+1)}
\end{aligned}
$$

We put $n=2$ in equation (44)

$$
y_{4}(x)=\pi^{2} S^{-1}\left[u^{2 \alpha} S\left\{A_{2}\right\}\right]
$$

Substituting equation (45) in (48) we get

$$
\begin{aligned}
y_{4}(x) & =\pi^{2} S^{-1}\left[u^{2 \alpha} S\left\{y_{2}(x) e^{y_{0}(x)}+\frac{y_{1}^{2}(x)}{2 !} e^{y_{0}(x)}\right\}\right] \\
& =\pi^{2} S^{-1}\left[u^{2 \alpha} S\left\{\frac{\pi^{2} x^{2 \alpha}}{\Gamma(2 \alpha+1)} e^{0}+\frac{\left(\frac{\pi x^{\alpha}}{\Gamma(\alpha+1)}\right)^{2}}{2 !} e^{0}\right\}\right] \\
& =\pi^{2} S^{-1}\left[u^{2 \alpha} S\left\{\frac{\pi^{2} x^{2 \alpha}}{\Gamma(2 \alpha+1)} 1+\frac{\pi^{2} x^{2 \alpha}}{2 !(\Gamma(\alpha+1))^{2}} 1\right\}\right] \\
& =\pi^{2} S^{-1}\left[u^{2 \alpha} \pi^{2} \frac{\left[2(\Gamma(\alpha+1))^{2}+\Gamma(2 \alpha+1)\right]}{2 \Gamma(2 \alpha+1)(\Gamma(\alpha+1))^{2}} S\left\{x^{2 \alpha}\right\}\right] \\
& =\pi^{4} \frac{\left[2(\Gamma(\alpha+1))^{2}+\Gamma(2 \alpha+1)\right]}{2 \Gamma(2 \alpha+1)(\Gamma(\alpha+1))^{2}} S^{-1}\left[u^{2 \alpha} u^{2 \alpha} \Gamma(2 \alpha+1)\right] \\
& =\pi^{4} \frac{\left[2(\Gamma(\alpha+1))^{2}+\Gamma(2 \alpha+1)\right]}{2(\Gamma(\alpha+1))^{2}} S^{-1}\left[u^{4 \alpha}\right] \\
& =\pi^{4} \frac{\left[2(\Gamma(\alpha+1))^{2}+\Gamma(2 \alpha+1)\right]}{2(\Gamma(\alpha+1))^{2}} \frac{x^{4 \alpha}}{\Gamma(4 \alpha+1)} \\
y_{4}(x) & =\frac{\left[2(\Gamma(\alpha+1))^{2}+\Gamma(2 \alpha+1)\right]}{2(\Gamma(\alpha+1))^{2} \Gamma(4 \alpha+1)} \pi^{4} x^{4 \alpha}
\end{aligned}
$$

The series solution is given by

$$
\begin{aligned}
y(x) & =y_{0}(x)+y_{1}(x)+y_{2}(x)+y_{3}(x)+\cdots \\
& =0+\frac{\pi x^{\alpha}}{\Gamma(\alpha+1)}+\frac{\pi^{2} x^{2 \alpha}}{\Gamma(2 \alpha+1)}+\frac{\pi^{3} x^{3 \alpha}}{\Gamma(3 \alpha+1)}+\frac{\left[2(\Gamma(\alpha+1))^{2}+\Gamma(2 \alpha+1)\right]}{2(\Gamma(\alpha+1))^{2} \Gamma(4 \alpha+1)} \pi^{4} x^{4 \alpha}+\cdots
\end{aligned}
$$

In particular case $\alpha=1$ then we get

$$
y(x)=\pi x+\frac{(\pi x)^{2}}{2 !}+\frac{(\pi x)^{3}}{3 !}+\frac{2(\pi x)^{4}}{4 !}+\cdots
$$

Table 3 shows that approximate solutions of $y(t)$ for (40) obtained for different values of $\alpha$ using the Sumudu decomposition method and DTM [15]. We can observe the numerical results in Table 3, it is clear that the approximate solutions of SDM are in best agreement 
with approximate solutions of DTM. Those solutions are constantly based on the Caputo fractional derivative. According to Taylors Theorem and its corollary, the obtained Taylor series is convergent about $\mathrm{a}=0$.

Fig. 3 shows the behaviour of approximate solutions versus exact solution. The nonlinear differential equation plots non-linear curves for various approximate values $\alpha=0.5,0.6,0.7,0.8,0.9$ and exact value $\alpha=1$. All non-linear curves of approximate solutions are very close to non-linear curve of exact solution.

Table 3. The comparisons of SDM and DTM for various values of $\alpha$. (for $\mathrm{x}=0.1$ and $\mathrm{N}=\mathrm{n}=5$ ).

\begin{tabular}{ccc}
\hline$\alpha$ & SDM & DTM [15] \\
\hline 0.5 & 2.8463 & 2.8288 \\
0.6 & 1.7418 & 1.7500 \\
0.7 & 1.0985 & 1.1107 \\
0.8 & 0.7495 & 0.7598 \\
0.9 & 0.5194 & 0.5270 \\
\hline
\end{tabular}

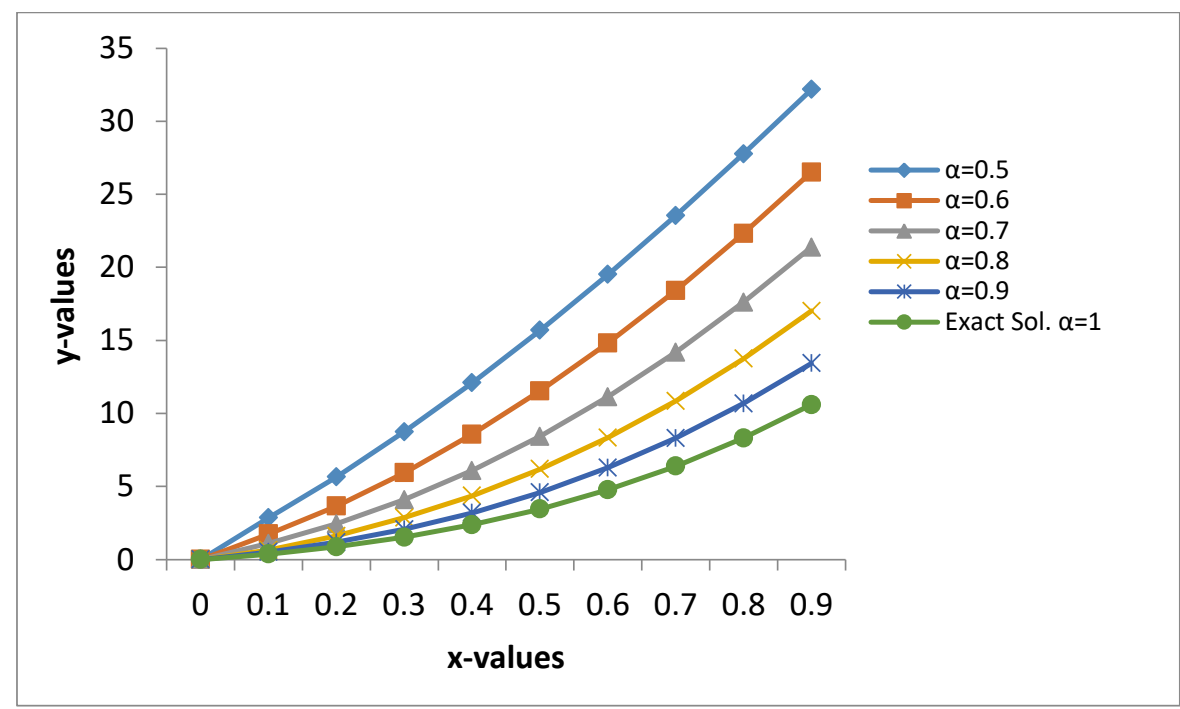

Fig. 3. The behavior of approximate solutions for the various values of $\alpha$ versus exact solution.

\subsection{Example}

Consider initial value Bratu-type problem

$$
D^{2 \alpha} y(x)+\pi^{2} e^{-y(x)}=0, \quad 0<\alpha \leq 1, \quad 0<x<1,
$$

Subject to initial condition

$$
y(0)=0, y^{\alpha}(0)=\pi
$$


The exact solution is given by $y(x)=\ln (1-\sin (\pi x))$ for $\alpha=1$.

For approximate solution, we apply Sumudu transform to both side of equation (49)

$$
\begin{aligned}
& S\left\{D^{2 \alpha} y(x)\right\}+\pi^{2} S\left\{e^{-y(x)}\right\}=0 \\
& S\left\{D^{2 \alpha} y(x)\right\}=-\pi^{2} S\left\{e^{-y(x)}\right\}
\end{aligned}
$$

By using definition 2.5 and initial condition (50) we have:

$$
\begin{aligned}
& \frac{Y(u)}{u^{2 \alpha}}-\frac{y(0)}{u^{2 \alpha-0}}-\frac{y^{\alpha}(0)}{u^{2 \alpha-\alpha}}=-\pi^{2} S\left\{e^{-y(x)}\right\} \\
& \frac{Y(u)}{u^{2 \alpha}}-\frac{\pi}{u^{\alpha}}=-\pi^{2} S\left\{e^{-y(x)}\right\} \\
& Y(u)=\pi u^{\alpha}-\pi^{2} u^{2 \alpha} S\left\{e^{-y(x)}\right\} \\
& S\{y(x)\}=\pi u^{\alpha}-\pi^{2} u^{2 \alpha} S\left\{e^{-y(x)}\right\}
\end{aligned}
$$

Further we apply inverse Sumudu transform to (51) to get

$$
\begin{aligned}
y(x) & =S^{-1}\left[\pi u^{\alpha}-\pi^{2} u^{2 \alpha} S\left\{e^{-y(x)}\right\}\right], \\
& =\pi S^{-1}\left[u^{\alpha}\right]-\pi^{2} S^{-1}\left[u^{2 \alpha} S\left\{e^{-y(x)}\right\}\right]
\end{aligned}
$$

Applying the Sumudu transform to initial condition yields $y_{0}(0)=0$. Considering $y_{1}(x)$ from source term and prescribed initial conditions

$$
\begin{aligned}
& y_{1}(x)=\pi S^{-1}\left[u^{\alpha}\right]=\frac{\pi x^{\alpha}}{\Gamma(\alpha+1)} \\
& y_{n+2}(x)=-\pi^{2} S^{-1}\left[u^{2 \alpha} S\left\{A_{n}\right\}\right]
\end{aligned}
$$

In this example, the nonlinear term is $N=e^{-y(x)}$

From equation (14) we have

$$
\begin{aligned}
& A_{0}=e^{-y_{0}(x)} \\
& A_{1}=-y_{1}(x) e^{-y_{0}(x)} \\
& A_{2}=-y_{2}(x) e^{-y_{0}(x)}+\frac{y_{1}^{2}(x)}{2 !} e^{-y_{0}(x)}
\end{aligned}
$$

We put $n=0$ in equation (53)

$$
y_{2}(x)=-\pi^{2} S^{-1}\left[u^{2 \alpha} S\left\{A_{0}\right\}\right]
$$

Substituting equation (54) in (55) we get

$$
\begin{aligned}
y_{2}(x) & =-\pi^{2} S^{-1}\left[u^{2 \alpha} S\left\{e^{-y_{0}(x)}\right\}\right] \\
& =-\pi^{2} S^{-1}\left[u^{2 \alpha} S\left\{e^{0}\right\}\right] \\
& =-\pi^{2} S^{-1}\left[u^{2 \alpha} S\{1\}\right] \\
& =-\pi^{2} S^{-1}\left[u^{2 \alpha}\right] \text { where } S\{1\}=1 \\
y_{2}(x) & =-\frac{\pi^{2} x^{2 \alpha}}{\Gamma(2 \alpha+1)}
\end{aligned}
$$


We put $n=1$ in equation (53)

$$
y_{3}(x)=-\pi^{2} S^{-1}\left[u^{2 \alpha} S\left\{A_{1}\right\}\right]
$$

Substituting equation (54) in (56) we get

$$
\begin{aligned}
y_{3}(x) & =-\pi^{2} S^{-1}\left[u^{2 \alpha} S\left\{-y_{1}(x) e^{-y_{0}(x)}\right\}\right] \\
& =-\pi^{2} S^{-1}\left[u^{2 \alpha} S\left\{-\frac{\pi x^{\alpha}}{\Gamma(\alpha+1)} e^{-y_{0}(x)}\right\}\right] \\
& =-\pi^{2} S^{-1}\left[u^{2 \alpha} S\left\{-\frac{\pi x^{\alpha}}{\Gamma(\alpha+1)} e^{0}\right\}\right] \\
& =-\pi^{2} S^{-1}\left[u^{2 \alpha} S\left\{-\frac{\pi x^{\alpha}}{\Gamma(\alpha+1)} 1\right\}\right] \\
& =-\pi^{2} S^{-1}\left[-\frac{u^{2 \alpha} \pi}{\Gamma(\alpha+1)} S\left\{x^{\alpha}\right\}\right] \\
& =-\pi^{2} S^{-1}\left[-\frac{u^{2 \alpha} \pi}{\Gamma(\alpha+1)} u^{\alpha} \Gamma(\alpha+1)\right] \\
& =\pi^{3} S^{-1}\left[u^{3 \alpha}\right] \\
y_{3}(x) & =\frac{\pi^{3} x^{3 \alpha}}{\Gamma(3 \alpha+1)}
\end{aligned}
$$

We put $n=2$ in equation (53)

$$
y_{4}(x)=-\pi^{2} S^{-1}\left[u^{2 \alpha} S\left\{A_{2}\right\}\right]
$$

Substituting equation (54) in (57) we get

$$
\begin{aligned}
y_{3}(x) & =-\pi^{2} S^{-1}\left[u^{2 \alpha} S\left\{-y_{2}(x) e^{-y_{0}(x)}+\frac{y_{1}^{2}(x)}{2 !} e^{-y_{0}(x)}\right\}\right] \\
& =-\pi^{2} S^{-1}\left[u^{2 \alpha} S\left\{\frac{\pi^{2} x^{2 \alpha}}{\Gamma(2 \alpha+1)} e^{0}+\frac{\left(\frac{\pi x^{\alpha}}{\Gamma(\alpha+1)}\right)^{2}}{2 !} e^{0}\right\}\right] \\
& =-\pi^{2} S^{-1}\left[u^{2 \alpha} S\left\{\frac{\pi^{2} x^{2 \alpha}}{\Gamma(2 \alpha+1)} 1+\frac{\pi^{2} x^{2 \alpha}}{2 !(\Gamma(\alpha+1))^{2}} 1\right\}\right] \\
& =-\pi^{2} S^{-1}\left[u^{2 \alpha} \pi^{2} \frac{\left[2(\Gamma(\alpha+1))^{2}+\Gamma(2 \alpha+1)\right]}{2 \Gamma(2 \alpha+1)(\Gamma(\alpha+1))^{2}} S\left\{x^{2 \alpha}\right\}\right] \\
& =-\pi^{4} \frac{\left[2(\Gamma(\alpha+1))^{2}+\Gamma(2 \alpha+1)\right]}{2 \Gamma(2 \alpha+1)(\Gamma(\alpha+1))^{2}} S^{-1}\left[u^{2 \alpha} u^{2 \alpha} \Gamma(2 \alpha+1)\right] \\
& =-\pi^{4} \frac{\left[2(\Gamma(\alpha+1))^{2}+\Gamma(2 \alpha+1)\right]}{2(\Gamma(\alpha+1))^{2}} S^{-1}\left[u^{4 \alpha}\right] \\
& =-\pi^{4} \frac{\left[2(\Gamma(\alpha+1))^{2}+\Gamma(2 \alpha+1)\right]}{2(\Gamma(\alpha+1))^{2}} \frac{x^{4 \alpha}}{\Gamma(4 \alpha+1)} \\
y_{4}(x) & =-\frac{\left[2(\Gamma(\alpha+1))^{2}+\Gamma(2 \alpha+1)\right]}{2(\Gamma(\alpha+1))^{2} \Gamma(4 \alpha+1)} \pi^{4} x^{4 \alpha}
\end{aligned}
$$

The series solution is given by

$$
y(x)=y_{0}(x)+y_{1}(x)+y_{2}(x)+y_{3}(x) \ldots \ldots \ldots \ldots
$$




$$
=0+\frac{\pi x^{\alpha}}{\Gamma(\alpha+1)}-\frac{\pi^{2} x^{2 \alpha}}{\Gamma(2 \alpha+1)}+\frac{\pi^{3} x^{3 \alpha}}{\Gamma(3 \alpha+1)}-\frac{\left[2(\Gamma(\alpha+1))^{2}+\Gamma(2 \alpha+1)\right]}{2(\Gamma(\alpha+1))^{2} \Gamma(4 \alpha+1)} \pi^{4} x^{4 \alpha}+\cdots
$$

In particular case $\alpha=1$ then we get

$$
y(x)=\pi x-\frac{(\pi x)^{2}}{2 !}+\frac{(\pi x)^{3}}{3 !}-\frac{2(\pi x)^{4}}{4 !}+\cdots
$$

Table 4 shows that approximate solutions of y(t) for (49) obtained for different values of $\alpha$ using the Sumudu decomposition method and DTM [15]. We can observe the numerical results in Table 4, it is clear that the approximate solutions of SDM are in best agreement with approximate solutions of DTM. Those solutions are constantly based on the Caputo fractional derivative. According to Taylors Theorem and its corollary, the obtained Taylor series is convergent about $\mathrm{a}=0$.

Fig. 4 shows the behaviour of approximate solutions versus exact solution. The nonlinear differential equation plots non-linear curves for various approximate values $\alpha=0.5,0.6,0.7,0.8,0.9$ and exact value $\alpha=1$. All non-linear curves of approximate solutions are very close to non-linear curve of exact solution.

Table 4. The comparisons of SDM and DTM for various values of $\alpha$ (for $\mathrm{x}=0.1$ and $\mathrm{N}=\mathrm{n}=5$ ).

\begin{tabular}{ccc}
\hline$\alpha$ & SDM & DTM [15] \\
\hline 0.5 & 0.8719 & 1.0790 \\
0.6 & 0.6112 & 0.7398 \\
0.7 & 0.4638 & 0.5359 \\
0.8 & 0.4025 & 0.4366 \\
0.9 & 0.3328 & 0.3474 \\
\hline
\end{tabular}

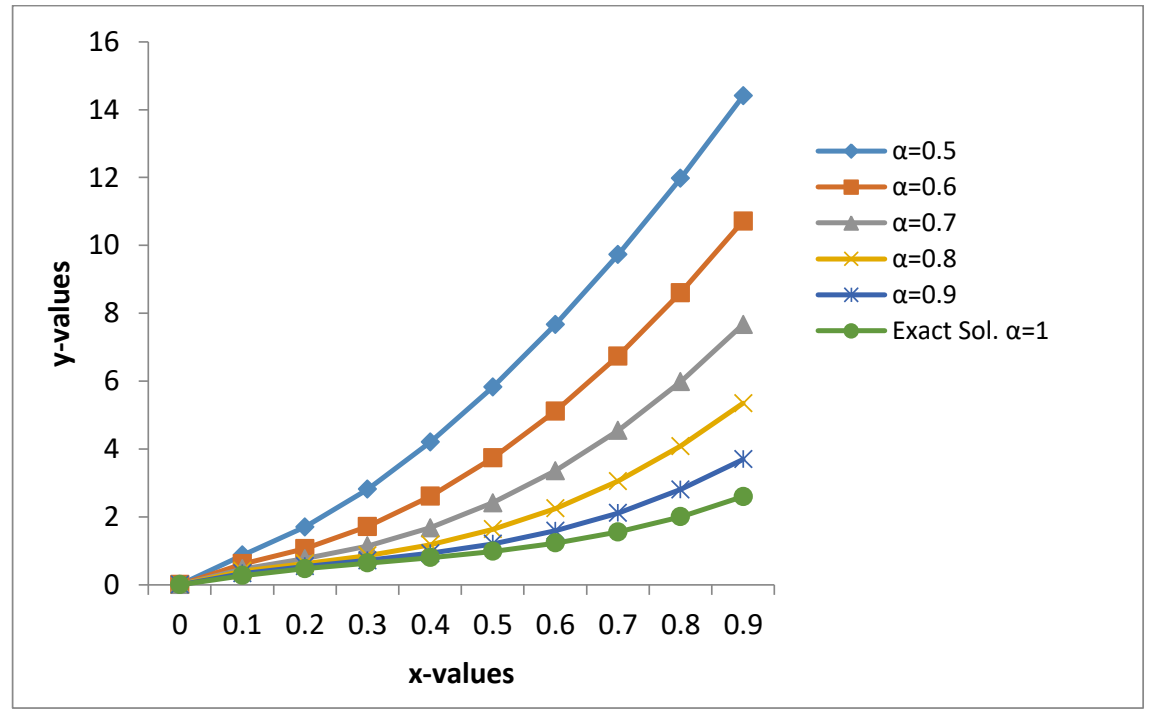

Fig. 4. The behavior of approximate solutions for the various values of $\alpha$ versus exact solution. 


\section{Conclusion}

In the present work, the approximate analytical solutions for nonlinear fractional Bratutype differential equations along with Caputo fractional derivative by using Sumudu decomposition method were procured. The obtained approximate solutions were written in a series form which converges rapidly. The approximate solutions were compared with DTM [15] and RKM [20]. We can observe that there exists obtained solution is an excellent agreement with already available solution. Thus, we say that this approach can be executed to solve the problems [FBDEs] effectively. With the help of obtained solutions, we observe that addition of one by one term in convergent series, the absolute errors and relative errors were decreased. We compared exact solution and approximate solutions by using graphical representation. This comparison suggests that our results are much better. We applied effectively the recurrence relation of Adomian polynomials for calculations of the non-linear terms. It is seen that the Sumudu decomposition method is implemented simply and effectively for fractional non-linear differential equations. Also, the SDM is applied for solution of non-linear problems without using He's polynomials, without identifying Lagrange's multiplier and without implementing quasilinearization technique. This is important advantage which is applicable for types of non-linear fractional differential equations. It is concluded that the present method gives good results. The proposed methodology can be implemented on other types of non-linear fractional differential equations and non-linear fractional partial differential equations.

\section{References}

1. I. Podlubny, Fractional Differential Equations (Academic press, San Diego, Calif, USA, 1999).

2. A. A. Kilbas, H. H. Srivastava, and J. J. Trujillo, Theory and Applications of Fractional Differential Equations (Elsevier, Amsterdam, The Netherlands, 2006).

3. R. L. Magin, Fractional Calculus in Bioengineering (Begell House, Conn, USA, Danbury, 2006).

4. J. W. Kirchner, C. Neal, and X. Feng, Nature 403, 524 (2000). https://doi.org/10.1038/35000537

5. R. Hilfer, Applications of Fractional Calculus in Physics (Word Scientific, Singapore, 2000). https://doi.org/10.1142/3779

6. H. Eltayeb and E. Abdeldaim, Res. Appl. Math. 1, 1 (2017). https://doi.org/10.11131/2017/101268

7. H. Khan, C. Tunc, R. A. Khan, A. G. Shirzoi, S. A. Gul, and A. Khan, Applicat. Appl. Math.: An Int. J. 13, 781 (2018).

8. M. M. Khader and K. M. Saad, Numerical Studies of the Fractional Korteweg-de Vries, Korteweg-de Vries-Burgers' and Burgers' Equations - Proceed. of the National Academy of Sciences (India, Sec. A Phys. Sci. 2020). https://doi.org/10.1007/s40010-020-00656-2

9. K. M. Saad, H. M. Srivastava, and J. F.Gómez-Aguilar, Chaos Solitons Fractals 132, 1 (2020). https://doi.org/10.1016/j.chaos.2019.109557

10. M. M. Khader and K. M. Saad, Int. J. Mod. Phys. C 31, 1 (2020). https://doi.org/10.1142/S0129183120500448

11. V. F. Morales-Delgado, J. F. Gómez-Aguilar, K. Saad, and R. F. Escobar Jiménez, Math. Methods Appl. Sci. 42, 1167 (2919). https://doi.org/10.1002/mma.5421 
12. K. M. Saad, E. H. F. AL-Shareef, A. K. Alomari, D. Baleanu, and J. F. Go'mez-Aguilar, Chinese J. Phys. 63, 149 (2020). https://doi.org/10.1016/j.cjph.2019.11.004

13. G. K. Watugala, Int. J. Math. Educ. Sci. Technol. 24, 35 (1993). https://doi.org/10.1080/0020739930240105

14. F. B. M. Belgacem and A. A. Karaballi, J. Appl. Math. Stoch. Anal. (2006). https://doi.org/10.1155/JAMSA/2006/91083

15. D. D. Demir and A. Zeybek, ITM Web Conf . 13, ID 01008 (2017). https://doi.org/10.1051/itmconf/20171301008

16. H. M. Baskonus, Z. Hammouch, T. Mekkaoui, and H. Bulut, Chaos in the Fractional Order Logistic Delay System, Circuit Realization and Synchronization - AIP Conf. Proc. (2016) 1738, p. 290005. https://doi.org/10.1063/1.4952077

17. A. Sadeghinia and P. Kumar, Int. J. Sci. Innov. Math. Res. 3, 14 (2015).

18. D. Kumar, J. Singh, and A. Kiliçman, Abstr. Appl. Anal. 2013 (2013). https://doi.org/10.1155/2013/608943

19. B. Ghazanfari, J. Math. Comput. Sci. 8, 236 (2014). https://doi.org/10.22436/jmcs.08.03.06

20. E. Babolian, S. Javadi, and E. Moradi, Math. Methods Appl. Sci. 39 (2015). https://doi.org/10.1002/mma.3588

21. K. Al-Khaled, Rom. J. Phys. 60, 1 (2015).

22. A. M. S. Mahdy and G. M. A. Marai, J. Abst. Comput. Math. 3, 42 (2018).

23. H. Z. Mjthap and S. N. Al-Azzawi Mixing, J. Interdisc. Math. 22, 1559 (2019). https://doi.org/10.1080/09720502.2019.1706858

24. I. Sumiati, E. R. Sukono, and A. T. Bon, IEOM Soc. Int. 23-26, 2409 (2019).

25. H. K. Jassima, M. G. Mohammed, and S. A. H. Khafif, Int. J. Adv. Appl. Math. Mech. 6, 64 (2019).

26. K. Saad and E. H. Al-Sharif, Adv. Differ. Equations 2017, ID 300 (2017). https://doi.org/10.1186/s13662-017-1358-0

27. K. M. Saad, A. A. AL-Shomrani, M. S. Mohamed, and X. J. Yang, Int. J. Open Problems Compt. Math. 9 (2016). https://doi.org/10.12816/0033919

28. M. Merdan, A. Yildirim, and A. Gökdoğan, Eng. Comput. 29, 766 (2012). https://doi.org/10.1108/02644401211257254

29. A. Elsaid, Appl. Math. Comput. 218, 6899 (2012). https://doi.org/10.1016/j.amc.2011.12.066

30. Y. Amer, A. Mahdy, and E. Youssef, Asian Res. J. Math. 7, 1 (2017). https://doi.org/10.9734/ARJOM/2017/32665 